\title{
Моделирование столкновения двух атомов над поверхностъю конденсированной фазы
}

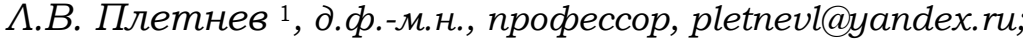 \\ C.A. Желтов 2, старший преподаватель, Zheltov.SA@tversu.ru \\ ${ }^{1}$ Тверской государственный технический университет, г. Тверь, 170026, Россия \\ 2 Тверской государственный университет, г. Тверь, 170100, Россия
}

Испарение вещества с поверхности конденсированной фазы представляет интерес как с практической, так и с теоретической точек зрения. Описать процесс вылета атомов с поверхности конденсированной фазы на микроскопическом уровне можно только с помощью метода молекулярной динамики, метода Монте-Карло или уравнения Больцмана. В связи с этим возникает вопрос о распределении столкновений атомов над поверхностью конденсированной фазы.

Для современной вычислительной техники моделирование испарения даже с микроскопических областей конденсированной фазы за малый промежуток времени - нереальная задача из-за большого объема вычислений. Существенную проблему представляет описание взаимодействия двух молекул в газовой фазе.

В статье анализируется проблема столкновения двух атомов над поверхностью конденсированной фазы после вылета с ее ограниченной области. В компьютерных экспериментах использовалась модель жестких сфер.

Моделирование методом Монте-Карло подразумевает статистическую обработку результатов достаточно большого числа экспериментов. Использование последовательных вычислений на стандартных CPU неэффективно с точки зрения времени выполнения программ. Последовательный алгоритм, реализующий моделирование столкновений 1 млн атомов, требует порядка 10 часов машинного времени. Для большей достоверности результатов желательно, чтобы в каждом эксперименте сталкивались как минимум 300 млн атомов. Алгоритм расчета обладает параллелизмом по данным и может быть адаптирован для параллельных вычислений на графических процессорах с архитектурой CUDA, что позволяет существенно снизить фактическое время выполнения программ и повысить достоверность результатов за счет увеличения количества разыгрываемых пар атомов.

Ключевые слова: параллельные вычисления, метод Монте-Карло, атом, столкновение, моделирование.

Оптимизация тепломассопереноса в технологических процессах связана с взаимодействием потока вещества со стенками систем [1-3]. Особый интерес представляет исследование процесса тепломассопереноса в наноразмерных системах [4-6], когда число Кнудсена больше единицы. На микроскопическом уровне испарение вещества с поверхности конденсированной фазы связано с взаимодействием вылетающих молекул с молекулами конденсированной фазы и с другими молекулами, вылетевшими с поверхности конденсированной фазы [7-9]. Возможен аналог образования слоя Кнудсена при испарении вещества [10-12]. В этом слое практически нет столкновений вылетающих с поверхности конденсированной фазы атомов друг с другом [13-15]. При слабоинтенсивном испарении вещества с поверхности конденсированной фазы атомы или молекулы могут пролететь достаточно большое расстояние до взаимного столкновения [16-18].
В данной работе на основе компьютерного эксперимента методом Монте-Карло проведен анализ распределений столкновений двух атомов над поверхностью, вылетевших с ограниченного участка поверхности конденсированной фазы. Метод Монте-Карло достаточно часто используется при численном моделировании различных физических систем. При этом достоверность результатов во многом обусловлена количеством сгенерированных и обработанных вариантов, что делает нецелесообразным использование последовательных вычислений на CPU из-за высоких затрат времени на проведение эксперимента.

Одним из способов повышения производительности является использование параллельных вычислений. Новый подход к рассматриваемой физической задаче и соответствующая модель процесса испарения потребовали разработки нового параллельного алгоритма и ПО. Одним из наиболее доступных вариантов орга- 
низации параллельных вычислений является использование GPU с архитектурой CUDA. Важным фактором при выборе вычислительной платформы послужило распараллеливание задачи по данным, что полностью соответствует специфике организации вычислений на графических процессорах. Это позволило создать программу, эффективно решающую задачу моделирования процесса испарения и получения распределений столкновений двух атомов, и получить достоверные статистические данные.

\section{Параллельный алгоритм моделирования столкновений двух атомов}

В процессе испарения с поверхности вылетает большое количество атомов с различными скоростями, часть которых сталкиваются друг с другом. Закон движения для каждого атома зависит только от начальных данных (координат на поверхности и скорости). Очевидно, что можно производить вычисления для нескольких пар атомов одновременно, то есть инструкции будут одинаковыми, а данные разными. Другими словами, задача относится к классу SIMD (Single Instruction stream/Multiple Data stream) и алгоритм может быть адаптирован к эффективным вычислениям на графических процессорах с технологией CUDA (Compute Unified Device Architecture) [19].

Блок-схема параллельного алгоритма моделирования столкновений атомов, вылетевших с поверхности конденсированной фазы, представлена на рисунке 1.

Программная реализация выполнена на языке $\mathrm{C}++$ с использованием набора расширений для графических процессоров Nvidia c apхитектурой CUDA. Для генерации значений случайных величин использовались библиотека CURAND и генераторы чисел, распределенных по нормальному и равномерному законам. Приведем пример заполнения массива случайными числами в параллельном режиме:

\#include <cuda.h>

\#include <curand.h>

...

cudaMalloc((void**)\& devX1, CHH *

sizeof(double));

cudaMalloc $\left(\left(\operatorname{void}^{* *}\right) \& \operatorname{devVX} 1, \mathrm{CHH} *\right.$

sizeof(double));

curandGenerator_t gen;

curandCreateGenerator(\&gen,

CURAND_RNG_PSEUDO_DEFAULT); curandSetPseudoRandomGeneratorSeed(gen, 1234ULL);
curandGenerateUniformDouble(gen, devX1, $\mathrm{CHH}$ );

curandGenerateNormalDouble(gen, devVX1,

$\mathrm{CHH}, \mathrm{m}$, sigma);

cudaMemcpy(ptrX1, devX1, CHH * sizeof(double), cudaMemcpyDeviceToHost);

cudaMemcpy(ptrVX1, devVX1, CHH *

sizeof(double), cudaMemcpyDeviceToHost);

Каждый компьютерный эксперимент проводился до тех пор, пока не разыгрывалось более 300 млн столкновений пар атомов. Так, 1200692724 столкновений произошло за 19.34 сек., 2101667707 - за 34.4 сек., 6303823665 - за 100.47 сек. Точность полученных результатов меньше нескольких сотых процента. Для каждой пары атомов разыгрывались их положения $\left(x_{1}, y_{1}\right)$ и $\left(x_{2}, y_{2}\right)$ на ограни-

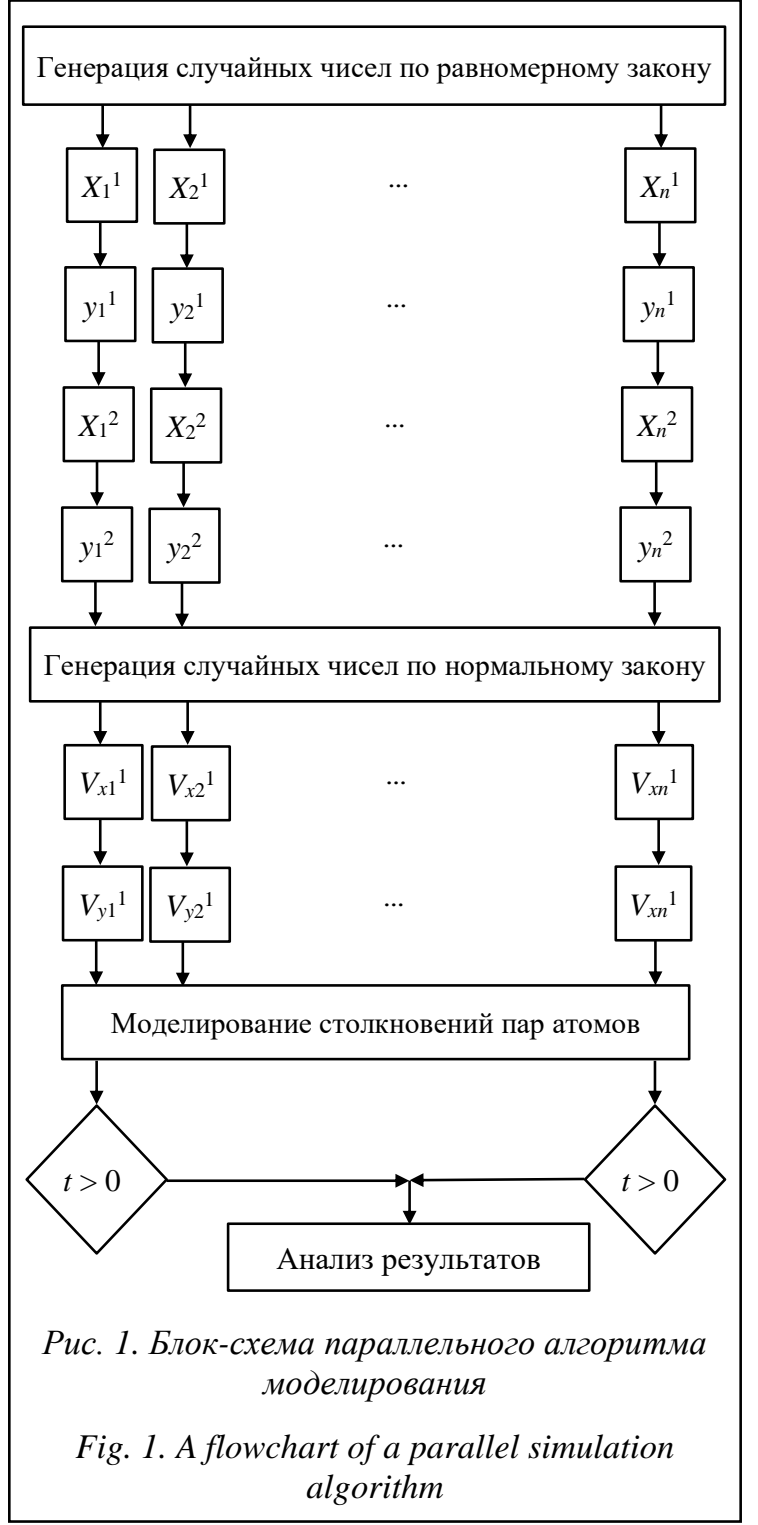




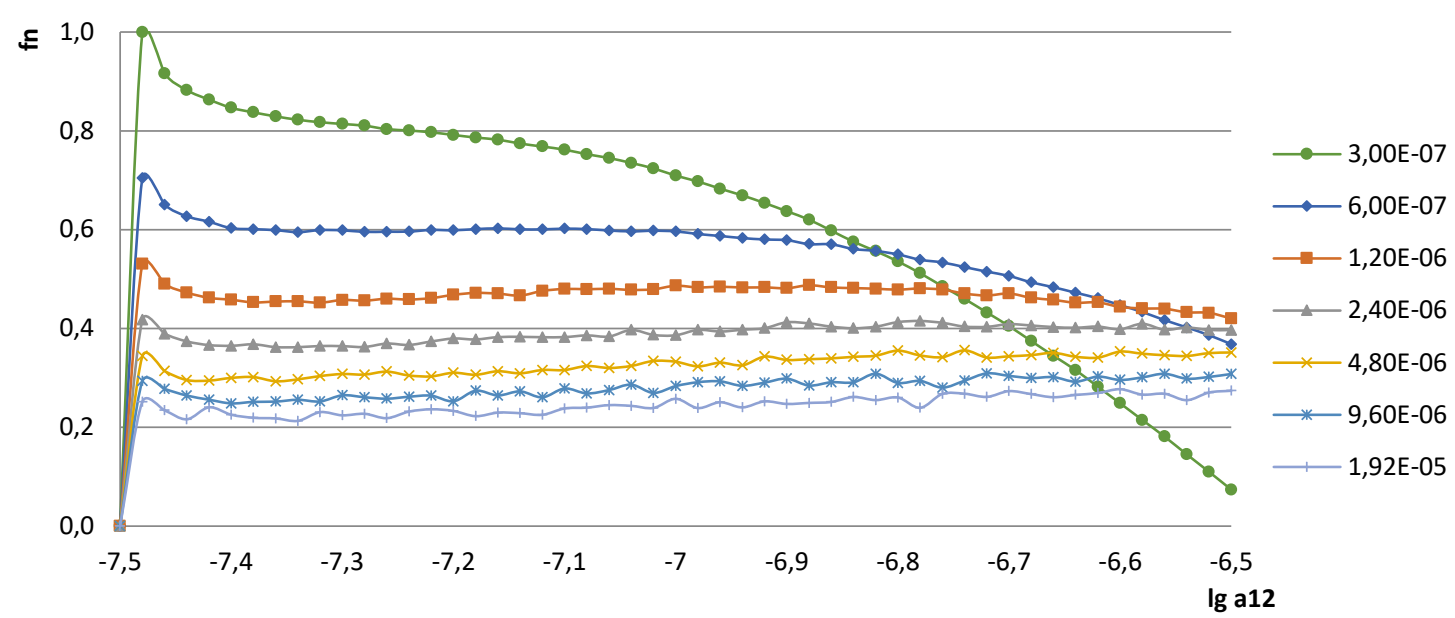

Рис. 2. Плотности распределений расстояний между столкнувиимися атомами при $T=50 \mathrm{~K}$

Fig. 2. Densities of distance distributions between colliding atoms at $T=50 \mathrm{~K}$

ченном участке поверхности конденсированной фазы со стороной $a=3 \cdot 10^{-9}$ м (в базовом варианте) и компоненты скоростей этих атомов $\left(v_{x 1}, v_{y 1}, v_{z 1}\right)$ и $\left(v_{x 2}, v_{y 2}, v_{z 2}\right)$ в плоскости $z=0$. В модели жестких сфер предполагалось, что атомы имеют вид сфер с радиусом $r=1,5 \cdot 10^{-10} \mathrm{M}$ и массой $m=40$ a.e.м.

Вычисления производились на гетерогенной платформе HybriLIT, состоящей из суперкомпьютера «Говорун» и учебно-тестового полигона HybriLIT. Данная платформа является частью многофункционального информационно-вычислительного комплекса лаборатории информационных технологий Объединенного института ядерных исследований, г. Дубна [20]. Для моделирования процесса столкновений атомов, вылетевших с поверхности конденсированной фазы, использовался ускоритель NVIDIA Tesla K80 (4 992 ядра CUDA на карте с двумя GPU, производительность в операциях с двойной точностью до 2,91 терафлопса) [21].

Как видно из результатов эксперимента, при значительном увеличении количества столкновений (соответственно, и разыгранных пар атомов, вылетевших с поверхности конденсированной фазы) фактическое время выполнения растет линейно. Адаптация алгоритма к параллельным вычислениям позволила смоделировать процесс вылета для увеличенных размеров области испарения и выявить зависимости от размера этой площади.

\section{Результаты расчетов}

В данной работе представлены результаты расчетов распределения столкновений двух атомов, вылетевших с ограниченной поверхности конденсированной фазы, в зависимости от размера области испарения $a$ и температуры поверхности конденсированной фазы $T$.

На рисунке 2 представлены результаты расчетов нормированных плотностей распределений расстояний между атомами на поверхности конденсированной фазы $a_{12}$, столкнувшимися над поверхностью. Минимальное расстояние равно диаметру атомов, а максимальное - положению атомов в противоположных углах области испарения.

Распределение средних расстояний между столкнувшимися атомами в зависимости от размера области испарения показано на рисунке 3. Установлено, что средние расстояния увеличиваются линейно с увеличением размера области испарения. Необходимо отметить: как плотности распределений, так и средние значения расстояний $a_{12}$ не зависят от тем-

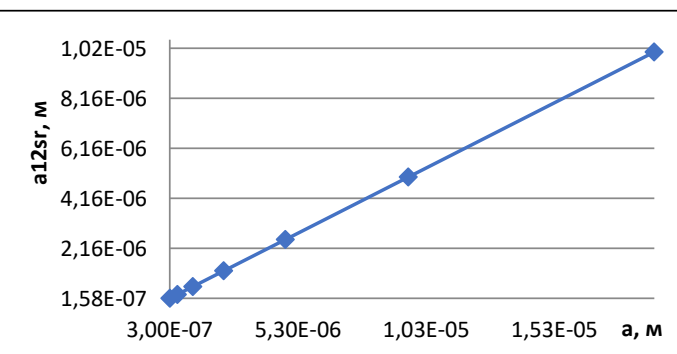

Рис. 3. Распределение средних значений расстояний между столкнувшимися атомами $a_{12}$ в конденсированной фазе

Fig. 3. Distribution of average distance values between colliding atoms $a_{12}$ in the condensed phase 


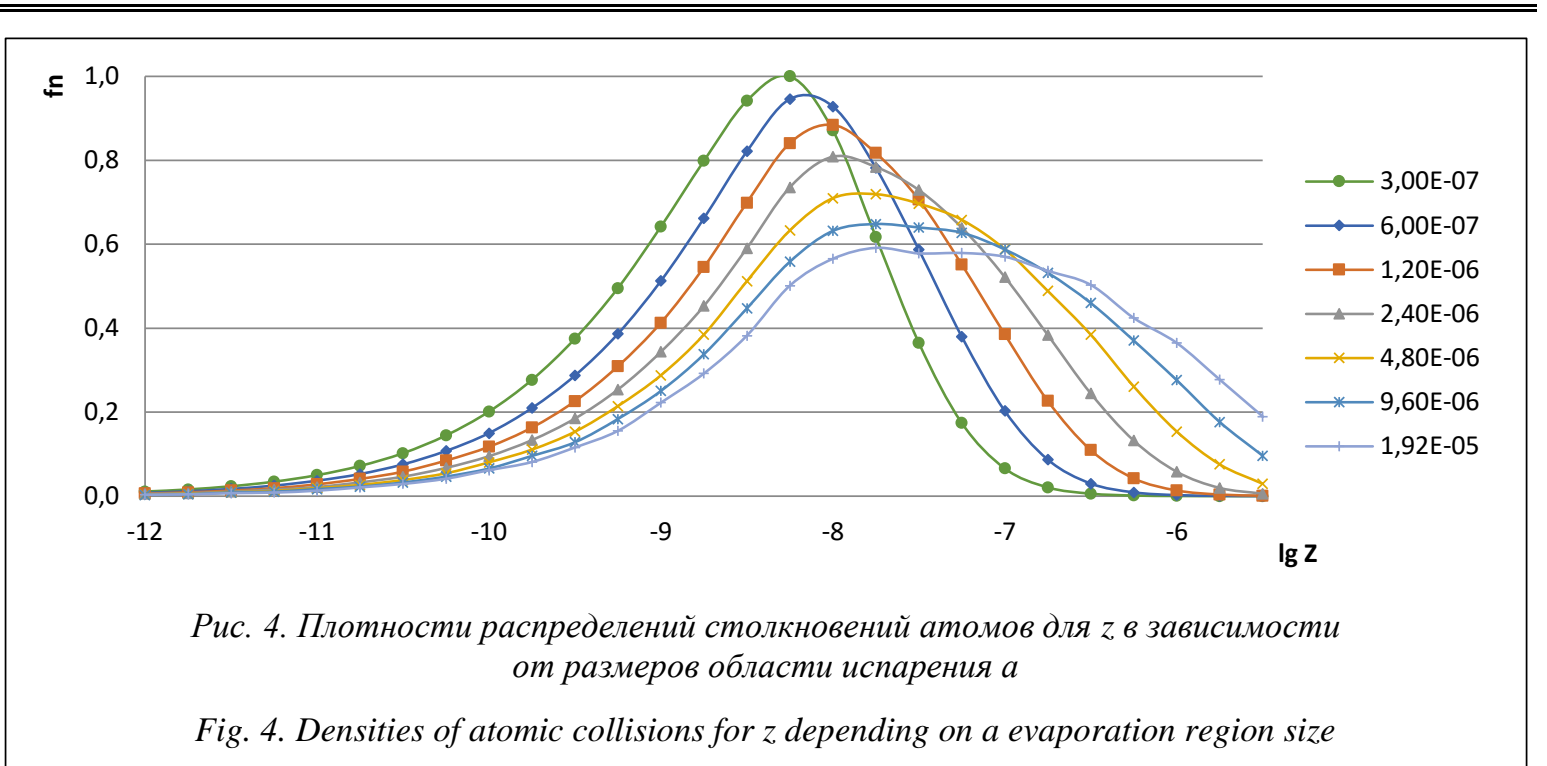

пературы конденсированной фазы $T$. Это можно объяснить тем, что положения атомов на поверхности конденсированной фазы разыгрывались с помощью датчика равномерно распределенных случайных величин. С физической точки зрения это означает, что интенсивность испарения уменьшается.

Проведенные компьютерные эксперименты показали, что плотности распределений столкновений над поверхностью конденсированной фазы $(z=0)$ не зависят от температуры конденсированной фазы $T$. Величина $z$ определялась как расстояние от поверхности конденсированной фазы до точки столкновения атомов. Это можно объяснить тем, что температура поверхности определяет средние скорости атомов, влияющие на время до столкновения атомов, но не на геометрию расстояния до столкновения.

Аналогичный вид имеет и плотность распределения длин пробега атомов до столкновения $l$. Данная величина определялась как квадратный корень из суммы квадратов проекций перемещений первого атома на оси координат.

Плотности распределений столкновений над поверхностью конденсированной фазы $z$ в зависимости от стороны области испарения $a$ отражены на рисунке 4. С увеличением размера области испарения плотности распределений и максимумы распределений смещаются в область больших значений $z$. Плотности распределений становятся более пологими, а максимумы распределений уменьшаются по величине. Это можно объяснить тем, что расстояния между сталкивающимися атомами на поверхности конденсированной фазы с увеличением области испарения растут.
Следует отметить, что кривые плотностей распределений для величины $l$ на графике (см. http://www.swsys.ru/uploaded/image/2020-2/202 0-2-dop/20.jpg) выглядят так же, как и для плотности распределения величины $z$. С увеличением размера области испарения $a$ увеличиваются не только расстояния до столкновения атомов над поверхностью, но и проекции пробегов атомов по осям $O x$ и $O y$. Вследствие этого соответствующие максимумы для величины $l$ сдвинуты вправо по отношению к максимумам величины $z$.

Представляет интерес получение распределений средних величин $z$ и $l$ в зависимости от размеров области испарения $a$. Установлено, что от температуры поверхности средние значения $z$ не зависят, так как они связаны с плотностями распределений $z$, которые также не зависят от температуры. Зависимость средних значений $z$ и $l$ от размеров области испарения $a$ показана на рисунке 5. Получены линейные зависимости от размеров области испарения $a$.

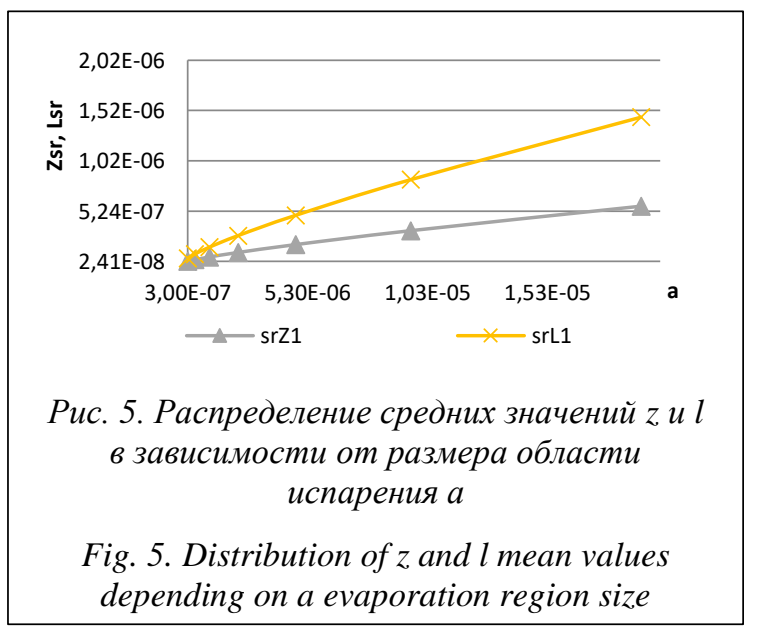


Необходимо отметить, что с увеличением области испарения более чем в 60 раз максимумы распределений, а соответственно, и средние значения, увеличиваются только на порядок.

\section{Заключение}

Полученные в компьютерных экспериментах результаты расчетов позволили установить закономерности распределений столкновений двух вылетевших атомов над поверхностью конденсированной фазы. Установлено, что плотности распределений имеют сложную структуру, которая только через средние значения может быть связана с определением слоя Кнудсена. Получить данные результаты удалось благодаря использованию параллельных вычислений на графических процессорах nVidia и адаптации алгоритма расчета столкновений атомов к архитектуре CUDA.

\section{Лumepamypa}

1. Lee J., Laoui T., Karnik R. Nanofluidic transport governed by the liquid/vapour interface. Nat. Nanotechnol., 2014, vol. 9, pp. 317-323. DOI: 10.1038/nnano.2014.28.

2. Li Y., Alibakhshi M.A., Zhao Y., Duan C. Exploring ultimate water capillary evaporation in nanoscale conduits. Nano Lett., 2017, vol. 17, pp. 4813-4819.

3. Wilke K.L., Barabadi B., Lu Z., Zhang T., Wang E.N. Parametric study of thin film evaporation from nanoporous membranes. Appl. Phys. Lett., 2017, vol. 111, iss. 17, art. 171603. DOI: 10.1063/1.4997945.

4. Xiao R., Maroo S.C., Wang E.N. Negative pressures in nanoporous membranes for thin film evaporation. Appl. Phys. Lett., 2013, vol. 102, iss. 12, art. 123103. DOI: 10.1063/1.4798243.

5. Lu Z., Narayanan S., Wang E.N. Modeling of evaporation from nanopores with nonequilibrium and nonlocal effects. Langmuir, 2015, vol. 31, pp. 9817-9824.

6. Frezzotti A. Boundary conditions at the vapor-liquid interface. Phys. Fluids, 2011, vol. 23, iss. 3, art. 030609. DOI: 10.1063/1.3567001.

7. Persad A.H., Ward C.A. Expressions for the evaporation and condensation coefficients in the HertzKnudsen relation. Chem. Rev., 2016, vol. 116, pp. 7727-7767.

8. Knudsen M. Die maximale verdampfungsgeschwindigkeit des quecksilbers. Ann. Phys., 1915, vol. 352 pp. 697-708.

9. Tcheremissine F.G. Solution to the Boltzmann kinetic equation for high-speed flows. Comput. Math. Phys., 2006, vol. 46, pp. 315-329.

10. Ishiyama T., Fujikawa S., Kurz T., Lauterborn W. Nonequilibrium kinetic boundary condition at the vapor-liquid interface of argon. Phys. Rev. E, 2013, vol. 88, iss. 4, art. 042406. DOI: 10.1103/PhysRevE.88. 042406.

11. Kon M., Kobayashi K., Watanabe M. Liquid temperature dependence of kinetic boundary condition at vapor-liquid interface. Int. J. Heat Mass Transfer, 2016, vol. 99, pp. 317-326.

12. Cheng S., Lechman J.B., Plimpton S.J., Grest G.S. Evaporation of Lennard-Jones fluids. J. Chem. Phys., 2011, vol. 134, iss. 22, art. 224704. DOI: 10.1063/1.3595260.

13. Kon M., Kobayashi K., Watanabe M. Method of determining kinetic boundary conditions in net evaporation/condensation. Phys Fluids, 2014, vol. 26, iss. 7, art. 072003. DOI: 10.1063/1.4890523.

14. Kon M., Kobayashi K., Watanabe M. Kinetic boundary condition in vapor-liquid two-phase system during unsteady net evaporation/condensation. Eur. J. Mech.-B/Fluids, 2017, vol. 64, pp. 81-92. DOI: 10.1016/j.euromechflu.2016.12.001.

15. Kobayashi K., Sasaki K., Kon M., Fujii H., Watanabe M. Kinetic boundary conditions for vapor-gas binary mixture. Microfluid Nanofluid, 2017, vol. 21. DOI: 10.1007/s10404-017-1887-6.

16. Уварова Л.А., Плетнев Л.В. Компьютерное моделирование столкновений частиц при вылете с поверхности конденсированной фазы // Тр. ИСА РАН. Динамика неоднородных систем. 2008. Т. 32. C. $131-139$.

17. Уварова Л.А., Плетнев Л.В. Распределения столкновений частиц при вылете с поверхности конденсированной фазы // Вестн. Поморского ун-та: Естественные науки. 2009. Т. 3. С. 102-107.

18. Плетнев Л.В. Распределения бинарных столкновений атомов при вылете с поверхности конденсированной фазы // Вестн. ТГТУ. 2011. Т. 17. Вып. 2. С. 520-524.

19. Желтов С.А. Особенности адаптации вычислительных алгоритмов к архитектуре CUDA // Maтематические методы управления: сб. науч. тр. ТГТУ. 2011. С. 33-36.

20. Гетерогенная платформа HybriLIT. URL: http://hlit.jinr.ru (дата обращения: 02.03.2020)

21. NVIDIA Tesla K80. URL: https://www.nvidia.com/ru-ru/data-center/tesla-k80 (дата обращения: 01.04.2020) 


\title{
Simulation of a collision of two atoms over a condensed phase surface
}

\author{
L.V. Pletnev ${ }^{1}$, Dr.Sc. (Physics and Mathematics), Professor, pletnevl@yandex.ru \\ S.A.Zheltov ${ }^{2}$, Senior Lecturer, Zheltov.SA@tversu.net \\ ${ }^{1}$ Tver State Technical University, Tver, 170100, Russian Federation \\ ${ }^{2}$ Tver State University, Tver, 170026, Russian Federation
}

\begin{abstract}
Evaporation of a substance from a condensed phase surface is of interest from both practical and theoretical points of view. It is possible to describe the process of departure of atoms from the condensed phase surface at the microscopic level only using the molecular dynamics method, the Monte Carlo method, or the Boltzmann equation. This raises the problem distributing collisions of atoms over the condensed phase surface.

Simulation of evaporation even from microscopic areas of the condensed phase in a short period of time is an unrealistic task for modern computer technology due to many calculations. The description of the interaction of two molecules in the gas phase is a significant problem.

The article analyzes the problem of collision of two atoms over the condensed phase surface after departure from its bounded areas. Computer experiments involved using a model of rigid spheres.

Monte Carlo simulation involves statistical processing of the results of a large number of experiments. Using sequential calculations on standard CPUs is inefficient in terms of program execution time. A sequential algorithm that implements simulation of collisions of 1000000 atoms requires about 10 hours of machine time. For greater reliability of the results, it is desirable that a minimum of 300000000 atoms collide in each experiment. The calculation algorithm has data parallelism and can be adapted for parallel calculations on graphics processors with the CUDA architecture, which makes it possible to significantly reduce the actual program execution time and increase the reliability of results by increasing the number of played pairs of atoms.
\end{abstract}

Keywords: parallel computing, Monte Carlo method, atom, collision, simulation.

\section{References}

1. Lee J., Laoui T., Karnik R. Nanofluidic transport governed by the liquid/vapour interface. Nat. Nanotechnol., 2014, vol. 9, pp. 317-323. DOI: 10.1038/nnano.2014.28.

2. Li Y., Alibakhshi M.A., Zhao Y., Duan C. Exploring ultimate water capillary evaporation in nanoscale conduits. Nano Lett., 2017, vol. 8, no. 17, pp. 4813-4819.

3. Wilke K.L., Barabadi B., Lu Z., Zhang T., Wang E.N. Parametric study of thin film evaporation from nanoporous membranes. Appl. Phys. Lett., 2017, vol. 111, iss. 17, art. 171603. DOI: 10.1063/1.4997945.

4. Xiao R., Maroo S.C., Wang E.N. Negative pressures in nanoporous membranes for thin film evaporation. Appl. Phys. Lett., 2013, vol. 102, iss. 12, art. 123103. DOI: 10.1063/1.4798243.

5. Lu Z., Narayanan S., Wang E.N. Modeling of evaporation from nanopores with nonequilibrium and nonlocal effects. Langmuir, 2015, vol. 31, pp. 9817-9824.

6. Frezzotti A. Boundary conditions at the vapor-liquid interface. Phys. Fluids, 2011, vol. 23, iss. 3, art. 030609. DOI: 10.1063/1.3567001.

7. Persad A.H., Ward C.A. Expressions for the evaporation and condensation coefficients in the HertzKnudsen relation. Chem. Rev., 2016, vol. 116, pp. 7727-7767.

8. Knudsen M. Die maximale verdampfungsgeschwindigkeit des quecksilbers. Ann. Phys., 1915, vol. 352, pp. 697-708.

9. Tcheremissine F.G. Solution to the Boltzmann kinetic equation for high-speed flows. Comput. Math. Phys., 2006, vol. 46, pp. 315-329.

10. Ishiyama T., Fujikawa S., Kurz T., Lauterborn W. Nonequilibrium kinetic boundary condition at the vapor-liquid interface of argon. Phys. Rev. E, 2013, vol. 88, iss. 4, art. 042406. DOI: 10.1103/PhysRevE.88. 042406.

11. Kon M., Kobayashi K., Watanabe M. Liquid temperature dependence of kinetic boundary condition at vapor-liquid interface. Int. J. Heat Mass Transfer, 2016, vol. 99, pp. 317-326.

12. Cheng S., Lechman J.B., Plimpton S.J., Grest G.S. Evaporation of Lennard-Jones fluids. J. Chem. Phys., 2011, vol. 134, iss. 22, art. 224704. DOI: 10.1063/1.3595260.

13. Kon M., Kobayashi K., Watanabe M. Method of determining kinetic boundary conditions in net evaporation/condensation. Phys Fluids, 2014, vol. 26, iss. 7, art. 072003. DOI: 10.1063/1.4890523. 
14. Kon M., Kobayashi K., Watanabe M. Kinetic boundary condition in vapor-liquid two-phase system during unsteady net evaporation/condensation. Eur. J. Mech.-B/Fluids, 2017, vol. 64, pp. 81-92. DOI: 10.1016/j.euromechflu.2016.12.001.

15. Kobayashi K., Sasaki K., Kon M., Fujii H., Watanabe M. Kinetic boundary conditions for vapor-gas binary mixture. Microfluid Nanofluid, 2017, vol. 21. DOI: 10.1007/s10404-017-1887-6.

16. Uvarova L.A., Pletnev L.V. Computer simulation of particle collisions during departure from the surface of the condensed phase. Proc. ISA RAS. Dynamics of Inhomogeneous Systems, 2008, vol. 32, pp. 131-139.

17. Uvarova L.A., Pletnev L.V. Distributions of particle collisions at departure from the surface of the condensed phase. Bull. Pomeranian Univ. Ser. Natural Sciences, 2009, vol. 3, pp. 102-107.

18. Pletnev L.V. Distributions of binary collisions of atoms at departure from the surface of the condensed phase. Bull. TSTU, 2011, vol. 17, iss. 2, pp. 520-524.

19. Zheltov S.A. Features of adaptation of computational algorithms to the CUDA architecture. Proc. TSTU. Mathematical Methods of Management, 2011, pp. 33-36.

20. Heterogeneous HybriLIT Platform. Avaible at: http://hlit.jinr.ru (accessed March 02, 2020).

21. NVIDIA Tesla K80. Avaible at: https://www.nvidia.com/ru-ru/data-center/tesla-k80 (accessed April 01, 2020).

\section{Для цитирования}

Пцетнев А.В., Желтов С.А. Моделирование столкновения двух атомов над поверхностью конденсированной фазы // Программные продукты и системы. 2020. T. 33. № 2. C. 297-303. DOI: 10.15827/0236-235X.130.297-303.

\section{For citation}

Pletnev L.V., Zheltov S.A. Simulation of a collision of two atoms over a condensed phase surface. Software \& Systems, 2020, vol. 33, no. 2, pp. 297-303 (in Russ.). DOI: 10.15827/0236-235X. 130.297-303. 